\title{
Inter- and intrarater reliability of goniometry and hand held dynamometry for patients with subacromial impingement syndrome
}

\author{
Georg Fieseler ${ }^{1, *}$, Kevin G. Laudner², Lars Irlenbusch ${ }^{3}$, Henrike Meyer ${ }^{3}$, Stephan Schulze ${ }^{3}$, Karl-Stefan Delank ${ }^{3}$, Souhail Hermassi ${ }^{4}$, \\ Thomas Bartels ${ }^{5}$, René Schwesig ${ }^{3}$ \\ 1 Division for Shoulder Surgery and Sports Orthopedics, Helios Clinic, Warburg/Westfalen, Germany \\ ${ }^{2}$ School of Kinesiology and Recreation, Illinois State University, Normal, IL, USA \\ ${ }^{3}$ Department of Orthopedic and Trauma Surgery, Martin-Luther-University Halle-Wittenberg, Halle (Saale), Germany \\ ${ }^{4}$ Tunisian Researches Laboratory "Sport Performance Optimization", Tunis, Tunisia \\ ${ }^{5}$ SportsClinic Halle, Center for Joint Surgery, Halle (Saale), Germany
}

The purpose of this study was to examine the intra- and interrater reliability of measuring shoulder range of motion (ROM) and strength among patients diagnosed with subacromial impingement syndrome (SAIS). Twenty-five patients (14 female patients; mean age, $60.4 \pm 7.84$ years) diagnosed with SAIS were assessed to determine the intrarater reliability for glenohumeral ROM. Twenty-five patients (16 female patients; mean age, $60.4 \pm 7.80$ years) and 76 asymptomatic volunteers ( 52 female volunteers; mean age, $29.4 \pm 14.1$ years) were assessed for interrater reliability. Dependent variables were active shoulder ROM and isometric strength. Intrarater reliability was fair-to-excellent for the SAIS patients (intraclass correlation coefficient [ICC], 0.52-0.97; standard error of measurement [SEM], $4.4^{\circ}-9.9^{\circ} \mathrm{N}$; coefficient of variation [CV], $7.1 \%-44.9 \%$ ). Based on the ICC, 11 of 12 parameters (92\%) dis-

\section{INTRODUCTION}

Before any measurements or assessments can be utilized for clinical or research related application, the reliability of these techniques must be determined (Koo and Li, 2016). Intraclass correlation coefficients (ICC) are calculated by mean squares obtained through an analysis of variance (Bartko, 1966). Currently, the ICC has been widely used in evaluation of interrater, intrarater or test-retest reliability (Portney and Watkins, 2000). These fundamental evaluations are the basis for clinical or research related assessments, because without them, we cannot conclude the reli- played an excellent reliability (ICC >0.75). The interrater reliability showed

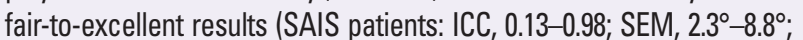
CV, 3.6\%-37.0\%; controls: ICC, 0.11-0.96; SEM, $3.0^{\circ}-35.4^{\circ}$; CV, $5.6 \%$ $26.4 \%$ ). In accordance with the intrarater reliability, glenohumeral adduction ROM was the only parameter with an ICC below 0.75 for both samples. Painful shoulder ROM in the SAIS patients showed no influence on the quality of reliability for measurement. Therefore, these protocols should be considered reliable assessment techniques in the prevention, diagnosis, and treatment of painful shoulder conditions such as SAIS.

Keywords: Hand-held dynamometry, Goniometry, Reliability, Shoulder
${ }^{*}$ Corresponding author: Georg Fieseler (10) https://orcid.org/0000-0003-2357-0447 Division for Shoulder Surgery and Sports Orthopedics, Helios Clinic,

Hueffertstrasse 50, 34414 Warburg/Westfalen, Germany

Tel: +49-5641-91-5502, Fax: +49-5641-91-5203

E-mail: georg.fieseler@helios-kliniken.de

Received: September 7, 2017 / Accepted: December 9, 2017 ability of any drawn conclusions (Koo and Li, 2016).

The measurement of flexibility and strength are critical aspects of a clinician's evaluation. Identifying aberrations in range of motion (ROM) or strength can assist in the prevention, diagnosis and rehabilitation of shoulder injuries (Kolber et al., 2012; Kolber and Hanney, 2012). Therefore, it is important for clinicians and researchers to utilize reliable and valid measurement techniques to objectively evaluate functional joint motion (Cools et al., 2014; Kolber et al., 2007, 2012; Kolber and Hanney, 2012). Goniometry has been widely used as the gold standard for clinical assessment because it is a cost effective method that can be used in al- 
most any setting. However, the tester is often required to use both hands, making stabilization of the joint more challenging or requiring the need for a second tester (Wilk et al., 2009). Manual muscle testing is often considered the gold standard among clinical techniques that assess strength (Schrama et al., 2014). Although isokinetic dynamometry provides a more sophisticated analysis, the upper extremity rarely produces isokinetic muscle actions during daily activities (Schrama et al., 2014). Subsequently, isokinetic testing is often not cost effective (Stark et al., 2011). Previous research has shown that testing with an isokinetic device has a strong correlation with a handheld dynamometer (HHD), indicating that this device is a valid tool for measuring upper extremity muscular strength (Stark et al., 2011). However, the HHD is limited by the strength and stabilisation capabilities of the examiner administering the test (Bohannon, 1986; Wang et al., 2002).

Subacromial impingement syndrome (SAIS) has been noted to be one of the most common shoulder injuries (Lewis, 2009). Patients with SAIS often present with various symptoms including altered muscular strength and ROM. More specifically, these patients may report with decreased glenohumeral internal and external rotation (ER) ROM (Tyler et al., 1999) as well as decreased muscular activity (Roddey et al., 2005) or rotator cuff strength (Leroux et al., 2015). Because of these alterations the assessment of ROM and strength among SAIS patients are considered to be critical aspects when assessing shoulder function (Cools et al., 2014). Therefore, the purpose of this study was to determine if there is any influence on inter- and intrarater reliability when using goniometry or a HHD to measure glenohumeral active ROM and isometric strength among SAIS patients.

\section{MATERIALS AND METHODS}

\section{Design}

This study used a descriptive design in a laboratory setting. Criteria for inclusion were the primary onset of SAIS of one shoulder with clinically pathological functional tests, positive radiological signs (i.e., pathological acromion types), and reduction of the acromiohumeral index and the acromiohumeral distance. For anamnesis there must have been no trauma, no prior conservative therapy or surgery, and no clinical or ultrasound signs of rotator cuff lesions. Only one shoulder was affected and each participant was screened by a medical doctor to rule out pre-existent neck and elbow pathologies. Subjects were excluded based on the following criteria: history of trauma, complaints of pain for 6 months or longer, preexistent shoulder surgeries, any kind of prior therapy, bilateral problems or neurological deficits incompliance to follow study protocol. Asymptomatic subjects were voluntarily recruited from a local Medical School. Each asymptomatic subject was clinically assessed for any neck, shoulder or elbow injuries. These control subjects were excluded if any persistent or recurrent pain or limited ROM was identified.

\section{Subjects}

Twenty-five patients ( 14 female, 11 male patients; age, $60.4 \pm 7.84$ years; height, $1.67 \pm 0.08 \mathrm{~m}$; mass, $76.2 \pm 16.6 \mathrm{~kg}$; body mass index, $27.0 \pm 4.61 \mathrm{~kg} / \mathrm{m}^{2}$ ) from an outpatient clinic for shoulder surgery (Division of the University Clinic) participated after random inclusion following written informed consent.

Twenty-five patients (16 female, 9 male patients; age, $60.4 \pm 7.80$ years; height, $1.67 \pm 0.09 \mathrm{~m}$; mass, $76.3 \pm 16.7 \mathrm{~kg}$; body mass index, $\left.27.0 \pm 4.58 \mathrm{~kg} / \mathrm{m}^{2}\right)$ from an outpatient clinic for shoulder surgery (Division of the University Clinic) were randomly included following written informed consent. Seventy-six asymptomatic volunteers from the Medical School ( 52 female, 24 male volunteers; age, $29.4 \pm 14.1$ years; height, $1.72 \pm 0.08 \mathrm{~m}$; mass, $70.6 \pm 14.3$ $\mathrm{kg}$; body mass index, $23.9 \pm 4.45 \mathrm{~kg} / \mathrm{m}$ ) participated in this study following written informed consent.

\section{Measurements}

Shoulder flexibility was measured using a standard goniometer, which has a reported accuracy within $1^{\circ}$ and a range of $180^{\circ}$. Isometric muscle strength was measured using the IsoForceControl $\mathrm{EVO}_{2}$ hand-held dynamometer (Medical Device Solutions AG, Oberburg, Switzerland), which can measure loads up to $400 \mathrm{~N}$.

All participants completed a standardized 2-min warm-up, which consisted of shoulder motions in all planes of movement. The glenohumeral active ROM and isometric strength assessments for all groups consisted of flexion, extension, internal rotation (IR), ER, abduction and adduction. One (intrarater) and two (interrater) experienced examiners completed all ROM and isometric strength measurements with support from a research assistant. The order of testing was not randomized and consisted of three trials for each measurement with one week between the two testing sessions. No subjects participated in any sport related activities during the testing period (Fieseler et al., 2015a, 2015b, 2015c).

\section{ROM measurements}

Participants were supine for all active glenohumeral ROM assessments. Their shoulders were placed into $90^{\circ}$ of shoulder abduction, $90^{\circ}$ of elbow flexion and a neutral wrist position (Fieseler 
et al., 2015a, 2015b, 2015c). Similar to the methodology used by Wilk et al. (2009) one investigator applied stabilization of the scapula to prevent any accessory movement (Fieseler et al., 2015a, 2015b, 2015c; Wilk et al., 2009). For rotational ROM the fulcrum of the goniometer was placed over the lateral epicondyle of the humerus, the stationary arm was vertical and the moveable arm in line with the styloid process of the ulna (Cools et al., 2014). For measurement of shoulder flexion and extension, subjects were asked to move to the lateral edge of the examination table to provide free range of movement forward and backward without destabilizing the scapula from the underground.

\section{Strength measurements}

Participants were supine for all isometric glenohumeral strength measurements with their test shoulder in $90^{\circ}$ of abduction, $90^{\circ}$ of elbow flexion, and a neutral wrist position. The investigator then provided stabilization of the scapula. Each subject held the HHD strap and provided an isometric contraction into either an internal or ER direction while a counter force was applied by the investigator. All isometric contractions were held for $10 \mathrm{sec}$ with a total of 3 trials per motion. The average of the 3 trials was then used for statistical analysis (Fieseler et al., 2015a, 2015b, 2015c).

\section{Statistical analysis}

Descriptive statistics for all participants were calculated. Dependent variables consisted of active ROM and isometric strength. A general linear model was used to identify any differences be- tween examinations (one and two) or investigators (one and two).

Test-retest reliability was assessed using ICC and standard error of measurement (SEM) (Schrama et al., 2014). Interpretation of ICC values was based on guidelines provided by Portney and Watkins (2000), where a value above 0.75 was classified as good or excellent reliability, while those below 0.75 are indicative of poor to moderate reliability (Portney and Watkins, 2000). SEM was reported in conjunction with the ICC's using the formula: SEM = standard deviation $\sqrt{1}-\mathrm{r}$ (Koo and Li, 2016; Portney and Watkins, 2000). 95\% limits of agreement (LOA) were calculated using the formula: $95 \% \mathrm{LOA}=$ mean difference $\pm 2 \mathrm{SD}$ ( $\mathrm{Koo}$ and $\mathrm{Li}$, 2016; Portney and Watkins, 2000). The coefficient of variation (CV) was derived from log-transformed data (Hopker et al., 2010). The $95 \%$ confidence intervals were also calculated for each CV and ICC. Finally, between tests differences were determined using one-way analysis of variance. IBM SPSS Statistics ver. 25.0 (IBM Co., Armonk, NY, USA) was utilized for all analyses.

\section{RESULTS}

Intrarater reliability for the SAIS group can be viewed in Table 1. Interrater reliability for the SAIS group can be found in Table 2, while Table 3 shows the interrater reliability for the control group. The intrarater reliability for painful shoulder varied from 0.52 for adduction ROM to 0.97 for IR strength. The SEM ranged from $4.36^{\circ}$ for extension ROM to $9.86 \mathrm{~N}$ for adduction strength. The CV moved between $7.1 \%$ for flexion ROM and $44.9 \%$ for adduc-

Table 1. Comparison of intrarater reliability measurements in patients with SAIS

\begin{tabular}{lrrrrr}
\hline Test & Session 1 & Session 2 & ICC (95\% CI) & SEM & CV (\%) (95\% CI) \\
\hline IR strength (N) & $61.5 . \pm 26.5$ & $60.1 . \pm 29.3$ & $0.97^{*}(0.92-0.99)$ & 4.83 & $14.3(11.8-22.0)$ \\
ER strength (N) & $50.5 . \pm 26.1$ & $53.5 . \pm 31.3$ & $0.90^{*}(0.78-0.96)$ & 9.08 & $21.7(18.5-35.3)$ \\
Flex strength (N) & $46.3 . \pm 18.3$ & $46.2 . \pm 17.3$ & $0.94^{*}(0.86-0.97)$ & 4.36 & $14.6(12.1-22.6)$ \\
Ext strength (N) & $69.8 . \pm 36.6$ & $64.2 . \pm 28.4$ & $0.94^{*}(0.86-0.98)$ & 7.96 & $15.3(12.7-23.7)$ \\
Abd strength (N) & $40.5 \pm 20.5$ & $38.3 . \pm 20.3$ & $0.94^{*}(0.86-0.97)$ & 5.00 & $21.7(18.5-35.3)$ \\
Add strength (N) & $52.2 . \pm 25.0$ & $54.8 . \pm 29.7$ & $0.87^{*}(0.70-0.94)$ & 9.86 & $26.0(22.5-43.6)$ \\
IR ROM $\left(^{\circ}\right)$ & $51.9 . \pm 14.0$ & $43.9 . \pm 20.3$ & $0.79^{*}(0.44-0.91)$ & 7.86 & $39.8(36.4-73.9)$ \\
ER ROM $\left(^{\circ}\right)$ & $65.2 . \pm 21.4$ & $59.4 . \pm 27.9$ & $0.94^{*}(0.83-0.97)$ & 6.04 & $24.5(21.1-40.6)$ \\
Flex ROM $\left(^{\circ}\right)$ & $162.3 . \pm 20.5$ & $158.4 . \pm 23.9$ & $0.88^{*}(0.74-0.95)$ & 7.69 & $7.1(5.7-10.4)$ \\
Ext ROM $\left(^{\circ}\right)$ & $60.6 . \pm 8.56$ & $62.5 . \pm 9.36$ & $0.76^{*}(0.46-0.89)$ & 4.39 & $9.4(7.6-14.0)$ \\
Abd ROM $\left(^{\circ}\right)$ & $127.1 . \pm 26.5$ & $127.4 . \pm 23.8$ & $0.89^{*}(0.74-0.95)$ & 8.34 & $9.6(7.8-14.3)$ \\
Add ROM $\left(^{\circ}\right)$ & $32.9 . \pm 9.84$ & $32.3 . \pm 15.6$ & $0.52(0-0.79)$ & 8.81 & $44.9(42.0-86.8)$ \\
\hline
\end{tabular}

Values are presented as mean \pm standard deviation.

SAIS, subacromial impingement syndrome; ICC, intrarater coefficient correlation; Cl, confidence interval; SEM, standard error of measurement; CV, coefficient of variation; IR, internal rotation; ER, external rotation; Flex, flexion; Ext, extension; Abd, abduction; Add, adduction; ROM, range of motion.

${ }^{*}$ ICC $>0.75$ highlighted in bold. 
Table 2. Comparison of interrater reliability measurements in patients with SAIS

\begin{tabular}{|c|c|c|c|c|c|}
\hline Test & Tester 1 & Tester 2 & ICC (95\% CI) & SEM & CV (95\% Cl) \\
\hline IR strength (N) & $60.7 \pm 26.3$ & $64.3 \pm 32.3$ & $0.93^{*}(0.85-0.97)$ & 7.75 & $17.3(14.5-27.3)$ \\
\hline ER strength (N) & $51.6 \pm 26.1$ & $57.4 \pm 31.4$ & $0.96^{*}(0.88-0.98)$ & 5.75 & $11.6(9.5-17.6)$ \\
\hline Flex strength (N) & $46.5 \pm 18.3$ & $51.0 \pm 24.1$ & $0.89^{*}(0.75-0.95)$ & 7.03 & $21.2(18.0-34.3)$ \\
\hline Ext stjrength (N) & $69.4 \pm 36.0$ & $69.0 \pm 37.1$ & $0.97^{*}(0.94-0.99)$ & 6.33 & $11.6(9.4-17.4)$ \\
\hline Abd strength (N) & $41.4 \pm 20.0$ & $39.4 \pm 21.6$ & $0.95^{*}(0.89-0.98)$ & 4.65 & $16.4(13.6-25.6)$ \\
\hline Add strength (N) & $52.1 \pm 25.0$ & $49.6 \pm 24.6$ & $0.98^{*}(0.95-0.99)$ & 3.51 & $12.9(10.6-19.6)$ \\
\hline $\operatorname{IRROM}\left({ }^{\circ}\right)$ & $50.9 \pm 15.4$ & $46.6 \pm 15.5$ & $0.86^{*}(0.67-0.94)$ & 5.78 & 18.1 (15.2-28.6) \\
\hline ER ROM $\left(^{\circ}\right)$ & $64.5 \pm 21.5$ & $65.1 \pm 21.7$ & $0.97^{*}(0.94-0.99)$ & 3.74 & $15.9(13.2-24.7)$ \\
\hline Flex ROM $\left(^{\circ}\right)$ & $161.7 \pm 20.3$ & $157.5 \pm 18.8$ & $0.94^{*}(0.85-0.98)$ & 4.79 & $3.6(2.9-5.2)$ \\
\hline Ext ROM $\left(^{\circ}\right)$ & $60.3 \pm 8.62$ & $60.4 \pm 8.51$ & $0.93^{*}(0.84-0.97)$ & 2.27 & $6.1(4.9-8.9)$ \\
\hline Abd ROM ( $\left.{ }^{\circ}\right)$ & $124.3 \pm 26.5$ & $129.2 \pm 23.3$ & $0.95^{*}(0.86-0.98)$ & 5.57 & $6.7(5.4-9.8)$ \\
\hline Add ROM $\left({ }^{\circ}\right)$ & $32.4 \pm 10.3$ & $41.4 \pm 8.48$ & $0.13(0-0.54)$ & 8.76 & $37.0(33.5-67.3)$ \\
\hline
\end{tabular}

Values are presented as mean \pm standard deviation.

SAIS, subacromial impingement syndrome; ICC, intrarater coefficient correlation; $\mathrm{Cl}$, confidence interval; SEM, standard error of measurement; $\mathrm{CV}$, coefficient of variation; IR, internal rotation; ER, external rotation; Flex, flexion; Ext, extension; Abd, abduction; Add, adduction; ROM, range of motion.

*ICC > 0.75 highlighted in bold.

Table 3. Comparison of interrater reliability measurements in healthy subjects

\begin{tabular}{lccccc}
\hline Test & Tester 1 & Tester 2 & ICC (95\% CI) & SEM & CV (95\% CI) \\
\hline IR strength (N) & $77.8 \pm 25.0$ & $74.9 \pm 19.5$ & $0.72^{*}(0.55-0.82)$ & 11.8 & $17.6(14.6-28.0)$ \\
ER strength (N) & $94.8 \pm 31.9$ & $94.7 \pm 30.3$ & $0.96^{*}(0.93-0.97)$ & 9.41 & $13.2(10.8-20.3)$ \\
Flex strength (N) & $70.2 \pm 18.3$ & $70.0 \pm 19.1$ & $0.95^{*}(0.92-0.97)$ & 4.18 & $13.2(10.8-20.4)$ \\
Ext strength (N) & $83.9 \pm 26.8$ & $82.6 \pm 28.0$ & $0.96^{*}(0.93-0.97)$ & 5.48 & $11.9(9.7-18.2)$ \\
Abd strength (N) & $63.7 \pm 19.1$ & $63.5 \pm 18.8$ & $0.91^{*}(0.85-0.94)$ & 5.69 & $21.6(18.3-35.4)$ \\
Add strength (N) & $71.4 \pm 23.6$ & $68.2 \pm 23.2$ & $0.93^{*}(0.89-0.96)$ & 6.19 & $13.1(10.7-20.1)$ \\
IR ROM $\left(^{\circ}\right)$ & $53.2 \pm 9.68$ & $51.4 \pm 9.19$ & $0.90^{*}(0.83-0.94)$ & 2.98 & $7.2(5.6-11.4)$ \\
ER ROM $\left(^{\circ}\right)$ & $84.4 \pm 14.5$ & $82.9 \pm 12.3$ & $0.91^{*}(0.86-0.94)$ & 4.02 & $5.6(4.3-8.8)$ \\
Flex ROM $\left(^{\circ}\right)$ & $171.9 \pm 9.84$ & $176.0 \pm 65.1$ & $0.11(0-0.45)$ & 35.4 & $26.4(21.9-48.5)$ \\
Ext ROM $\left(^{\circ}\right)$ & $59.9 \pm 6.53$ & $59.1 \pm 6.14$ & $0.68(0.49-0.80)$ & 3.58 & $11.4(8.9-18.6)$ \\
Abd ROM $\left(^{\circ}\right)$ & $167.0 \pm 17.6$ & $167.2 \pm 19.8$ & $0.86^{*}(0.77-0.91)$ & 7.00 & $9.9(7.7-16.0)$ \\
Add ROM $\left(^{\circ}\right)$ & $48.7 \pm 15.9$ & $45.5 \pm 9.27$ & $0.59(0.34-0.74)$ & 8.06 & $20.7(16.8-36.4)$ \\
\hline
\end{tabular}

Values are presented as mean \pm standard deviation.

ICC, intrarater coefficient correlation; Cl, confidence interval; SEM, standard error of measurement; CV, coefficient of variation; IR, internal rotation; ER, external rotation; Flex, flexion; Ext, extension; Abd, abduction; Add, adduction; ROM, range of motion.

${ }^{*}$ ICC $>0.75$ highlighted in bold.

tion strength. Based on the ICC, 11 of 12 parameters (92\%) displayed an excellent reliability (ICC $>0.75$ ).

Among both intrarater and interrater reliability $89 \%$ (32 of 36) of all shoulder parameters showed excellent reliability. The interrater reliability showed poor-to-excellent results (SAIS patients: ICC, $0.13-0.98$; SEM, $2.3^{\circ}-8.8^{\circ}$; CV, 3.6\%-37.0\%; healthy subjects: ICC, 0.11-0.96; SEM, 3.0-35.4\%; CV, 5.6\%-26.4\%). In accordance with the intrarater reliability, adduction ROM was the only parameter with an ICC below 0.75 for both samples. Additionally for healthy subjects, the flexion ROM of shoulder showed also a poor level of reliability (ICC, 0.11 ; SEM, $35.4^{\circ} ; \mathrm{CV}, 26.4 \%$ ).
During intrarater reliability measurements the SAIS patients showed the largest LOA for adduction strength (38.0 N; upper limit, 35.4; lower limit, -40.6). The smallest LOA was found for extension ROM (15.8 $8^{\circ}$; upper limit, $13.8^{\circ}$; lower limit, $\left.-17.8^{\circ}\right)$. For interrater reliability among SAIS patients the largest LOA was detected for IR strength $(29.7 \mathrm{~N}$; upper limit, $11.3 \mathrm{~N}$; lower limit, $-18.4 \mathrm{~N})$ and the smallest LOA for extension ROM $\left(9.0^{\circ}\right.$; upper limit, $4.4^{\circ}$; lower limit, $\left.-4.7^{\circ}\right)$. The IR ROM was the parameter with the smallest LOA for healthy subjects $\left(14.1^{\circ}\right.$; upper limit, $9.4^{\circ}$; lower limit, $\left.-4.7^{\circ}\right)$. In comparison, flexion ROM showed the largest LOA for healthy individuals $\left(133.3^{\circ}\right.$; upper limit, $64.9^{\circ}$; 
lower limit, $\left.-68.4^{\circ}\right)$. Altogether, the limits of agreement for healthy subjects were surprisingly larger compared to the SAIS patients (mean LOA: $20.0^{\circ}$ vs. $39.6^{\circ}$ ).

\section{DISCUSSION}

This is the first study to investigate the parameters for intraand interrater reliability of measuring glenohumeral active ROM and isometric strength among patients diagnosed with painful SAIS and control participants. This study reported moderate-to-excellent intrarater reliability for all active shoulder ROM measurements. These results also showed good-to-excellent intrarater reliability for isometric shoulder strength tests in all ROM directions using a HHD. For interrater reliability all shoulder ROM and strength measurements showed excellent reliability with the exception of adduction ROM, which was poor. These results demonstrate the good reliability and accuracy of measuring glenohumeral ROM and strength among patients diagnosed with SAIS.

Cools et al. (2014) measured the reliability of passive shoulder ROM among individuals without any pain using two investigators during a single testing session. These authors reported that placing patients in different positions and using different technical equipment, such as goniometers and inclinometers, may influence the outcomes for both internal and ER (Cools et al., 2014). Conversely, other results (Kolber et al., 2007, 2012) have reported interchangeable results when using a goniometer and digital inclinometer. Due to such discrepancies in previous research it is important to identify reliable and accurate clinical methods for assessing shoulder ROM and strength. This is evident among SAIS patients who often present with less internal and ER ROM compared to controls (Tyler et al., 1999). Due to the common occurrence of posterior shoulder tightness among these patients it is critical to identify accurate methods for assessing ROM. The findings of the current study demonstrate that using a goniometer and proper stabilization is a reliable method for measuring active ROM among patients with SAIS. This technique also showed similar reliability to those of Kolber et al. (2007, 2012), Kolber and Hanney (2012), and our Fieseler et al. (2015a, 2015b, 2015c) who measured active shoulder ROM and others who have investigated passive ROM among asymptomatic individuals (Cools et al., 2014).

There is a plethora of research reporting on the reliability of using a HHD for measuring shoulder strength among asymptomatic individuals. This reliability has been investigated among varying scenarios such as repeated use during a single session (Bohan- non, 1986), under different shoulder positions (Andersen et al., 2014), using different stabilization techniques (Kolber et al., 2007), using different dynamometer placements (McMahon et al., 1992), and tester strength (Wikholm and Bohannon, 1991) to name but a few. Much of this research has shown good reliability. One study (Gajdosik, 2005) reported that when assessing children between the ages of 28-50 months there is fair-to-excellent reliability. Similarly, other research has shown good reliability, however this finding was assessed in adults without any throwing experience (Wang et al., 2002). Conversely, Schrama et al. (2014) concluded that testing strength with a HHD was only acceptable for the elbow among asymptomatic individuals. There are even reliability reports for strength among varying pathologic individuals such as patients diagnosed with spinal cord injuries (May et al., 1997), cervical radiculopathy (Persson et al., 1997), chronic obstructive pulmonary disease (O'Shea et al., 2007), brachial plexus injuries (Tsai et al., 2015), and even Duchenne muscular dystrophy (Stuberg and Metcalf, 1988).

Despite SAIS being one of the most common shoulder disorders among individuals of all ages and activity levels (Lewis, 2009), no research has investigated the reliability of measuring isometric strength among this population. This void of knowledge is even more important when you consider that previous research has reported insufficient muscular activity or weakness of the rotator cuff among patients with SAIS. More specifically, Leroux et al. (2015) showed that SAIS patients have less internal and ER strength, as well as a less internal-external rotator ratio compared to controls. Furthermore, muscle activity among SAIS patients has been shown to be reduced during the first arc of motion and the inferior force vector, created by the infraspinatus and subscapularis, is less functional when compared to controls (Reddy et al., 2000). Due to these altered muscular activity and strength levels among SAIS patients it is important to understand the reliability of measuring this functional characteristic. Although, previous research has reported a large span of ICC grades for glenohumeral strength, especially among athletes and symptomatic patients (Schrama et al., 2014). The results of the current study are the first to investigate and report good reliability for measuring glenohumeral strength among SAIS patients. These findings emphasize the use of consistent and standardized protocols during assessment, such as subject positioning and stabilization.

As with any research there are some limitations worth noting from this study. End ROM measurements taken during active ROM tests are determined through subjective criteria. Similarly, during strength assessments the authors of this study had to as- 
sume that each participant was providing a maximum effort. Although our ROM techniques were found to be reliable, these measurements required two clinicians to be effective: one examiner to align the goniometer and an additional investigator to stabilize the scapula. This may prove problematic for some clinicians who do not often have access to an assistant during examinations. This study also consisted of only patients diagnosed with SAIS. Patients with other forms of shoulder, elbow, or spinal pathologies may present with varying results. Future studies should investigate the reliability for measuring flexibility and strength among a variety of symptomatic populations, such as SLAP lesions, internal impingement, and adhesive capsulitis.

This study demonstrated a clinically applicable and standardized protocol for determining glenohumeral ROM and strength. More specifically, these techniques revealed good-to-excellent intra- and interrater reliability for active shoulder ROM and isometric strength using a standard goniometer and HHD among SAIS patients. Interrater glenohumeral adduction ROM was the only characteristic found to have poor reliability. The positioning and stabilization techniques described in this study should be considered in the prevention, evaluation, and rehabilitation of patients diagnosed with SAIS. These findings may provide clinicians with the confidence for using similar techniques in the evaluation and treatment of patients with shoulder pain.

\section{CONFLICT OF INTEREST}

No potential conflict of interest relevant to this article was reported.

\section{REFERENCES}

Andersen KS, Christensen BH, Samani A, Madeleine P. Between-day reliability of a hand-held dynamometer and surface electromyography recordings during isometric submaximal contractions in different shoulder positions. J Electromyogr Kinesiol 2014;24:579-587.

Bartko JJ. The intraclass correlation coefficient as a measure of reliability. Psychol Rep 1966;19:3-11.

Bohannon RW. Test-retest reliability of hand-held dynamometry during a single session of strength assessment. Phys Ther 1986;66:206-209.

Cools AM, De Wilde L, Van Tongel A, Ceyssens C, Ryckewaert R, Cambier DC. Measuring shoulder external and internal rotation strength and range of motion: comprehensive intra-rater and inter-rater reliability study of several testing protocols. J Shoulder Elbow Surg 2014; 23:1454-1461.
Fieseler G, Jungermann P, Koke A, Irlenbusch L, Delank KS, Schwesig R. Glenohumeral range of motion (ROM) and isometric strength of professional team handball athletes, part III: changes over the playing season. Arch Orthop Trauma Surg 2015a;135:1691-1700.

Fieseler G, Jungermann P, Koke A, Irlenbusch L, Delank KS, Schwesig R. Range of motion and isometric strength of shoulder joints of team handball athletes during the playing season, Part II: changes after midseason. J Shoulder Elbow Surg 2015b;24:391-398.

Fieseler G, Molitor T, Irlenbusch L, Delank KS, Laudner KG, Hermassi S, Schwesig R. Intrarater reliability of goniometry and hand-held dynamometry for shoulder and elbow examinations in female team handball athletes and asymptomatic volunteers. Arch Orthop Trauma Surg 2015c;135:1719-1726.

Gajdosik CG. Ability of very young children to produce reliable isometric force measurements. Pediatr Phys Ther 2005;17:251-257.

Hopker J, Myers S, Jobson SA, Bruce W, Passfield L. Validity and reliability of the Wattbike cycle ergometer. Int J Sports Med 2010;31:731-736.

Kolber MJ, Beekhuizen K, Cheng MS, Fiebert IM. The reliability of handheld dynamometry in measuring isometric strength of the shoulder internal and external rotator musculature using a stabilization device. Physiother Theory Pract 2007;23:119-124.

Kolber MJ, Fuller C, Marshall J, Wright A, Hanney WJ. The reliability and concurrent validity of scapular plane shoulder elevation measurements using a digital inclinometer and goniometer. Physiother Theory Pract 2012;28:161-168.

Kolber MJ, Hanney WJ. The reliability and concurrent validity of shoulder mobility measurements using a digital inclinometer and goniometer: a technical report. Int J Sports Phys Ther 2012;7:306-313.

Koo TK, Li MY. A guideline of selecting and reporting intraclass correlation coefficients for reliability research. J Chiropr Med 2016;15:155-163.

Leroux T, Chahal J, Wasserstein D, Verma NN, Romeo AA. A systematic review and meta-analysis comparing clinical outcomes after concurrent rotator cuff repair and long head biceps tenodesis or tenotomy. Sports Health 2015;7:303-307.

Lewis JS. Rotator cuff tendinopathy/subacromial impingement syndrome: is it time for a new method of assessment? Br J Sports Med 2009;43:259264.

May LA, Burnham RS, Steadward RD. Assessment of isokinetic and handheld dynamometer measures of shoulder rotator strength among individuals with spinal cord injury. Arch Phys Med Rehabil 1997;78:251255.

McMahon LM, Burdett R, Whitney SL. Effects of muscle group and placement site on reliability of hand-held dynamometry strength measurements. J Orthop Sports Phys Ther 1992;15:236-242.

O'Shea SD, Taylor NF, Paratz JD. Measuring muscle strength for people 
with chronic obstructive pulmonary disease: retest reliability of handheld dynamometry. Arch Phys Med Rehabil 2007;88:32-36.

Persson LC, Moritz U, Brandt L, Carlsson CA. Cervical radiculopathy: pain, muscle weakness and sensory loss in patients with cervical radiculopathy treated with surgery, physiotherapy or cervical collar. A prospective, controlled study. Eur Spine J 1997;6:256-266.

Portney LG, Watkins MP. Foundations of clinical research: applications to practice. 2nd ed. Upper Saddle River (NJ): Prentice-Hall; 2000.

Reddy AS, Mohr KJ, Pink MM, Jobe FW. Electromyographic analysis of the deltoid and rotator cuff muscles in persons with subacromial impingement. J Shoulder Elbow Surg 2000;9:519-523.

Roddey TS, Cook KF, O'Malley KJ, Gartsman GM. The relationship among strength and mobility measures and self-report outcome scores in persons after rotator cuff repair surgery: impairment measures are not enough. J Shoulder Elbow Surg 2005;14(1 Suppl S):95S-98S.

Schrama PP, Stenneberg MS, Lucas C, van Trijffel E. Intraexaminer reliability of hand-held dynamometry in the upper extremity: a systematic review. Arch Phys Med Rehabil 2014;95:2444-2469.

Stark T, Walker B, Phillips JK, Fejer R, Beck R. Hand-held dynamometry correlation with the gold standard isokinetic dynamometry: a system- atic review. PM R 2011;3:472-479.

Stuberg WA, Metcalf WK. Reliability of quantitative muscle testing in healthy children and in children with Duchenne muscular dystrophy using a hand-held dynamometer. Phys Ther 1988;68:977-982.

Tsai YJ, Tu YK, Hsiao CK, Su FC. Within-session reliability and smallest real difference of muscle strength following nerve transfers in patients with brachial plexus injuries. J Hand Surg Am 2015;40:1196-1201.

Tyler TF, Roy T, Nicholas SJ, Gleim GW. Reliability and validity of a new method of measuring posterior shoulder tightness. J Orthop Sports Phys Ther 1999;29:262-269.

Wang CY, Olson SL, Protas EJ. Test-retest strength reliability: hand-held dynamometry in community-dwelling elderly fallers. Arch Phys Med Rehabil 2002;83:811-815.

Wikholm JB, Bohannon RW. Hand-held dynamometer measurements: tester strength makes a difference. J Orthop Sports Phys Ther 1991;13: 191-198.

Wilk KE, Reinold MM, Macrina LC, Porterfield R, Devine KM, Suarez K, Andrews JR. Glenohumeral internal rotation measurements differ depending on stabilization techniques. Sports Health 2009;1:131-136. 